\title{
Note sur la nidification dans le sol de deux Hoplitis du Maroc : Hoplitis (Anthocopa) zaianorum BENOIST 1927 et Hoplitis (Hoplitis) tenuiserrata BENOIST 1950 (Apoidea - Megachilidae - Osmiini)
}

\author{
par Gérard LE GoFF*
}

Résumé. Dans cette note sont décrits deux nids établis dans le sol par deux Hoplitis au Maroc : Hoplitis zaianorum BENOIST 1927 et Hoplitis tenuiserrata BENOIST 1950. Ces nids ont été collectés près du village de Tiout $\left(30,4209^{\circ} \mathrm{N}-8,6696^{\circ} \mathrm{O}\right)$.

Abstract. In this note are described two nests built in the soil by two Hoplitis in Marocco: Hoplitis zaianorum BENOIST 1927 and Hoplitis tenuiserrata BENOIST 1960. Those nests were collected near the Tiout village $\left(30,4209^{\circ} \mathrm{N}-8,6696^{\circ} \mathrm{W}\right)$.

Mots-clés : nids ; Megachilidae ; Osmiini ; Hoplitis zaianorum ; Hoplitis tenuiserrata.

\section{Introduction}

En mars - avril 2013, avec mes amis E. Dufrêne et N. Vereecken, nous avons effectué notre séjour de printemps dans le Sud marocain. À partir de Taroudannt, nous avons sillonné la région pour collecter des abeilles. Pour ma part, j'ai trouvé d'intéressantes espèces de Megachilidae dont de nombreuses Hoplitis.

C'est en prospectant un champ en bord de route, un peu avant le village de Tiout, que j'ai eu la chance de découvrir deux nids en construction et capturer leur fondatrice respective (figure 1).

* Gérard LE GOFF

44, rue Albert Malet, 76360 Barentin (F)

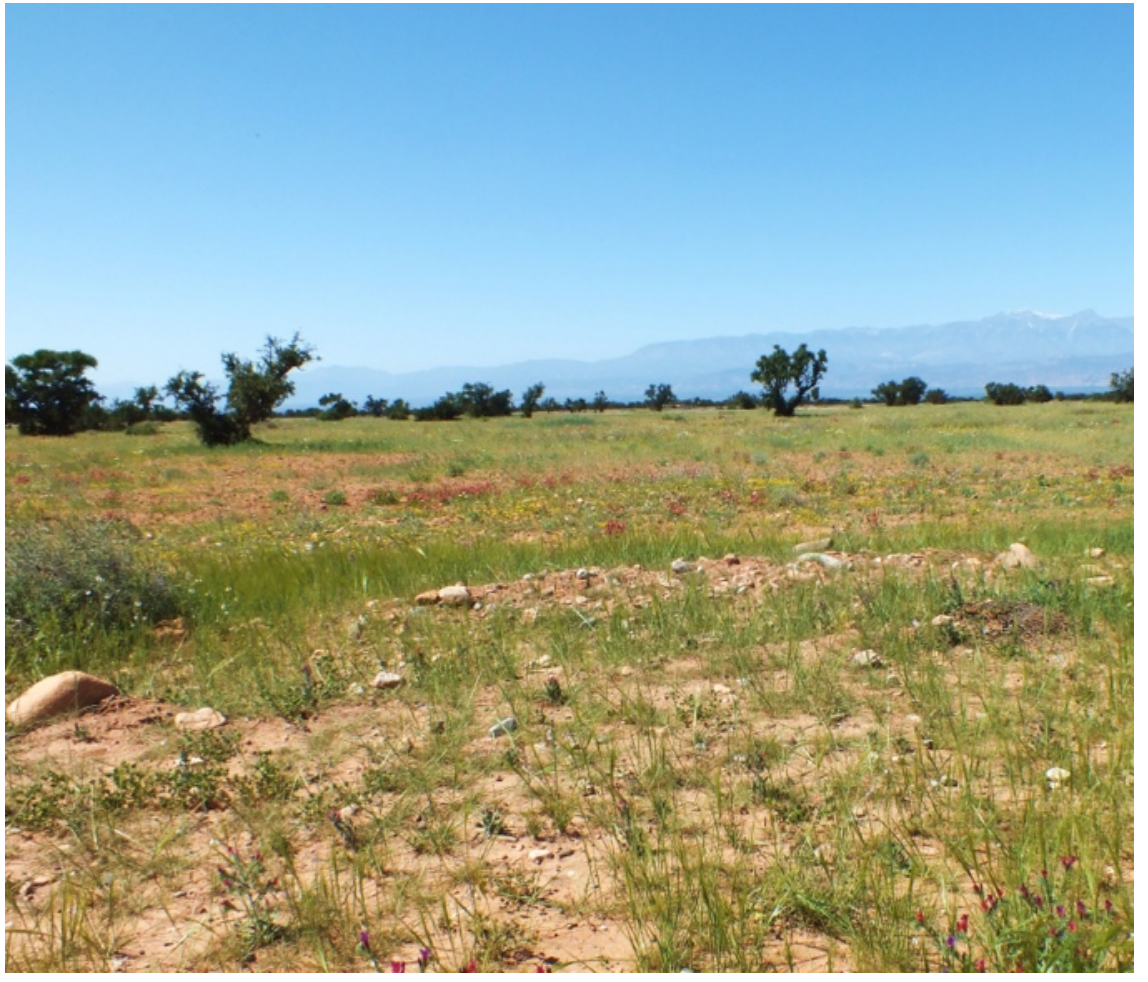

Figure 1. Site de collecte des deux nids dans la vallée du Souss, près du village de Tiout. Le champ est planté de blé très épars et de quelques arganiers, à de nombreux endroits le sol est à nu. Diverses plantes sont aussi présentes sur le site, comme de l'Echium plantagineum, du Lotus corniculatus, de la Centaurea solticialis, etc... Photo : Gérard Le Goff 


\section{Matériel}

Nid N597 - fondatrice : Hoplitis (Anthocopa) zaianorum f. ( $\left.\mathrm{N}^{\circ} 15023\right)$ - coll. Le Goff 02.IV-2013 - Tiout (Maroc) - 30,4209 ${ }^{\circ} \mathrm{N}$ $-8,6696^{\circ} \mathrm{O}$

Nid N596 - fondatrice : Hoplitis (Hoplitis) tenuiserrata f. ( $\left.\mathrm{N}^{\circ} 15022\right)$ - coll. Le Goff - 02IV-2013 - Tiout (Maroc) - 30,4209 ${ }^{\circ} \mathrm{N}-8,6696^{\circ} \mathrm{O}$

Hoplitis zaianorum est une abeille de taille moyenne, d'environ 9-10 $\mathrm{mm}$, à pilosité roussâtre et blanchâtre avec une brosse ventrale rousse. Dans le sous-genre Anthocopa, elle appartient au groupe " singularis" appelée auparavant "Glossosmia».

Hoplitis tenuiserrata est une plus petite abeille d'environ 7-8 $\mathrm{mm}$ à pilosité blanc roussâtre et blanche ; la brosse de récolte est blanche. Elle appartient au sous-genre Hoplitis s. str. et au petit groupe « monstrabilis ».

\section{Nidification d'Hoplitis (Anthocopa) zaianorum BENOIST. (N597)}

L'entrée du nid (figure 2a), de 4,5 $\mathrm{mm}$ de diamètre, se trouvait à l'abri d'une pierre. La galerie d'accès, légèrement plus large $(5 \mathrm{~mm})$ s'enfonce en biais. Elle est élargie dans sa partie antérieure, offrant un espace équivalent à une cellule. Ce vestibule est utile à la manœuvre et la garde. Des galeries secondaires également obliques mènent chacune à une cellule. Ce nid, de type " rayonnant " comprend 5 branches menant à 5 cellules. Quatre sont complètes; une est en construction. Les galeries divergentes sont relativement courtes et mesurent de 12 à $15 \mathrm{~mm}$. Elles sont comblées de terre après l'achèvement de la cellule terminale. Chaque cellule est ovoïde, longue de $10-11 \mathrm{~mm}$ et mesure $6 \mathrm{~mm}$ dans sa plus grande largeur.

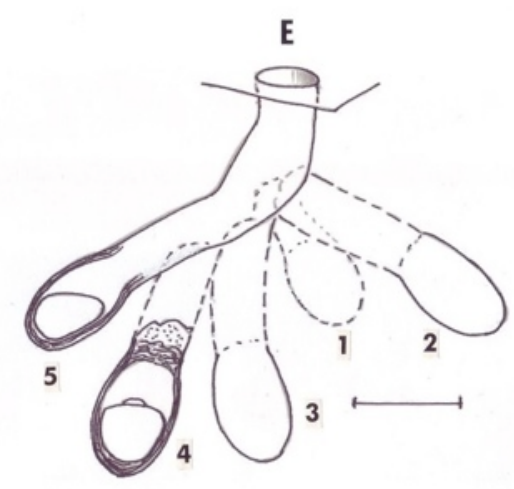

Figure 2a. Schéma du nid d'Hoplitis zaianorum (N597). Échelle $10 \mathrm{~mm}$.

Dessin : Gérard Le Goff
La loge est tapissée de découpes de pétales de Lotus corniculatus abondant en bordure de route et en plaques dans le champ. La pâtée pollinique sur laquelle l'œuf est pondu, occupe presque la moitié de l'espace cellulaire. Elle est élaborée à partir de pollen de Centaurée (très certainement C. solticialis présente plus à l'intérieur du champ). Cela m'a été confirmé par Andreas Müller à qui j'ai envoyé le contenu d'une des cellules pour examen (figure $2 b$ ).

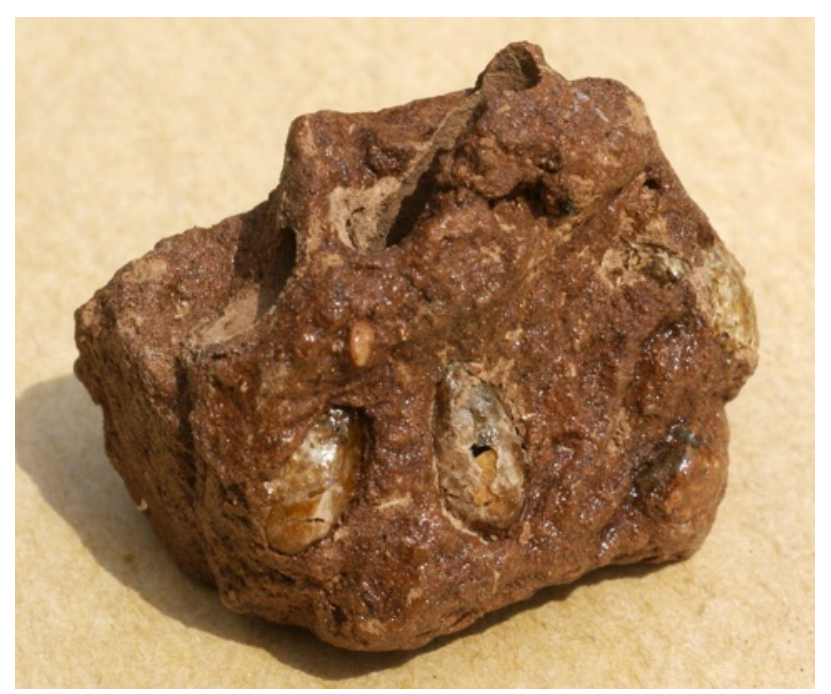

Figure 2b. Nid d'Hoplitis zaianorum ( $\mathrm{N}_{597}$ ) dégagé du bloc de terre. Le contenu de la cellule 2 a été examiné pour identifier le pollen. La cellule 5 , en construction, est ouverte pour en voir la structure. Photo : Gérard Le Goff

Cela conforte ses propres observations sur le type de pollen récolté par H. zaianorum. D'ailleurs, le groupe "singularis» est sans doute oligolectique sur les Carduoideae (MÜLLER, 2013). Dans les premières cellules, les larves sont déjà bien développées. Chaque cellule est close par un bouchon de fragments de pétales. La galerie d'accès est ensuite remplie de terre (venant sans doute du forage suivant). On peut supposer que le nid sera finalement obturé par un tampon de terre.

\section{Nidification d'Hoplitis (Hoplitis) tenuiserrata BENOIST. (N596)}

Ce deuxième nid était foré à environ $2 \mathrm{~m}$ de distance du précédent, avec son ouverture à découvert sur le sol nu à cet endroit. C'est un petit nid unicellulaire (figure $3 a$ ) avec une galerie d'accès verticale courte, de $8 \mathrm{~mm}$ de long et de $3,5 \mathrm{~mm}$ de large. Au bout se trouve une cellule ovale très arrondie de $7,5 \mathrm{~mm}$ de long et de $5 \mathrm{~mm}$ dans sa plus grande largeur. Les parois sont lissées et la pâtée pollinique est déposée à 
même le fond de la cellule. Cette réserve forme une boule qui occupe les $2 / 3$ de la loge (figure $3 b$ ). L'œuf est pondu à son sommet.

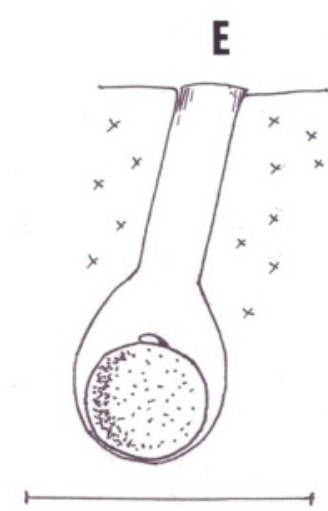

Figure зa. Schéma du nid d'Hoplitis tenuiserrata (N596).

Échelle $10 \mathrm{~mm}$.

Dessin : Gérard Le Goff

Le pollen provient du Lotier (Lotus corniculatus) cité précédemment. La collecte de ce pollen est caractéristique de ce petit groupe d'Hoplitis qui est certainement oligolectique sur Fabaceae avec une forte préférence pour les Loteae (ROZEN et al., 2009 - SÉDIVY et al., 2013a). La cellule sera cloisonnée de terre, ainsi que le nid.

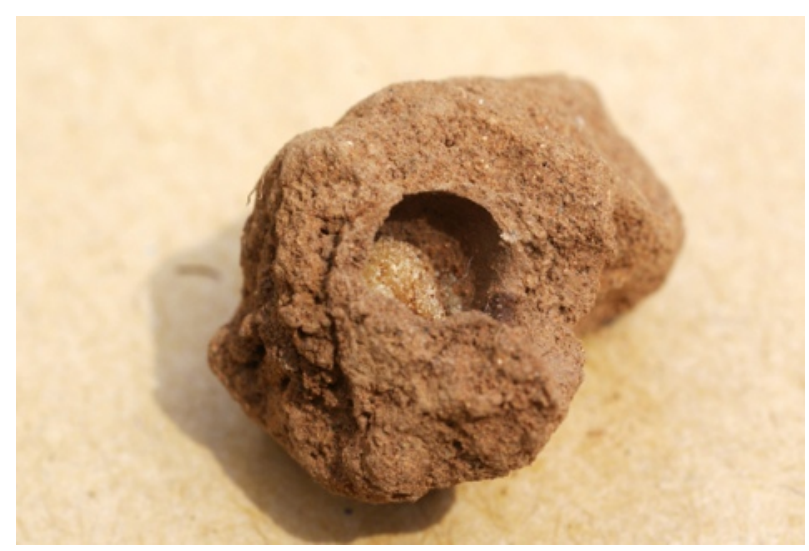

Figure 3 b. Nid d'Hoplitis tenuiserrata (596) après son ouverture : on voit bien la « boule » pollinique. Photo : Gérard Le Goff

\section{Remerciements}

Je tiens à exprimer toute ma gratitude à Andreas Müller, de l'ETH de Zürich, qui a bien voulu déterminer mes deux Hoplitis et également identifier le pollen utilisé par H. zaianorum.

\section{Références bibliographiques}

BENOIST R, 1927. Hyménoptères mellifères nouveaux du Maroc. Bulletin de la Société des Sciences Naturelles du Maroc (Rabat) 7, 169-174.

Müller A, 2013. Palaearctic Osmiine Bees, ETH Zürich, http://blogs.ethz.ch/osmiini

ROzEN JG et al., 2009. Biology of the bee Hoplitis (Hoplitis) monstrabilis Tkalcu and descriptions of its egg and larva (Megachilidae ; Megachilinae ; Osmiini). American Museum Novitates 3680, 1-22.

SÉDIVy C et $\boldsymbol{a l}$., 2013. Host range evolution in a selected group of osmiine bees (Hymenoptera ; Megachilidae) : the Boraginaceae - Fabaceae paradox. Biological Journal of the Linnean Society 108, 35-54.

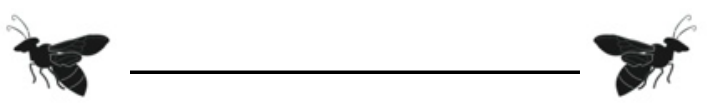

